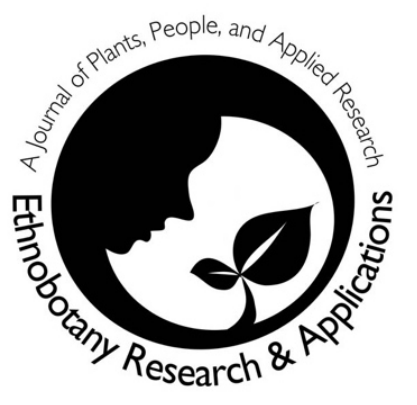

\title{
Food and culture: Cultural patterns related to food by indigenous communities in Kashmir - A Western Himalayan region
}

Musheerul Hassan, Umer Yaqoob, Shiekh Marifatul Haq, Hammad Ahmad Jan, Huma Habib, Saima Hamid, Fayaz A. Lone and Rainer W. Bussmann

\section{Correspondence}

Musheerul Hassan ${ }^{1}$, Umer Yaqoob²., Shiekh Marifatul Haq ${ }^{2 *}$, Hammad Ahmad Jan ${ }^{3}$, Saima Hamid ${ }^{5}$, Fayaz A. Lone ${ }^{6}$ and Rainer W. Bussmann ${ }^{7}$

${ }^{1}$ Clybay Research Private Limited, Bangalore, India - 560114

${ }^{2}$ Department of Botany, University of Kashmir, Hazratbal, Srinagar, J\&K- India-190006

${ }^{3}$ Department of Botany, University of Buner, Swari, Pakistan

${ }^{4}$ Islamia College of Science and Commerce, Srinagar, J\&K, India - 192302

${ }^{5}$ Centre of Research for Development/Environmental Sciences, Srinagar, Jammu and Kashmir, India - 190006

${ }^{6}$ Govt. Degree College (Women) Kupwara, J\&K, India - 193222

${ }^{7}$ Department of Ethnobotany, Institute of Botany, Ilia State University, 0105 Tbilisi, Georgia

${ }^{*}$ Corresponding Author: snaryan17@gmail.com

Ethnobotany Research \& Applications 22:44 (2021)

\section{Research}

\begin{abstract}
Background: Food and culture have both local and global implications. Through their distinct culinary habits, communities are linked to their culture. Foods can transmit a variety of cultural meanings in and of themselves. It describes the community's social standing, ethnicity, and income in addition to informing us about a specific event. A certain culture or sub-culture to which they belong passes down its food habits with great care.
\end{abstract}

Methods. Semi-structured questionnaires were used to collect information, whereas principal component analysis and Two-way cluster analysis was done to analyze the data using PAST software ver.3.14.

Results. The present study recorded 75 edible species used in cultural foods in the Kashmir valley. These species were grouped as animals $(\mathrm{N}=6)$, birds $(\mathrm{N}=12)$, fish $(\mathrm{N}=5)$, vegetables $(\mathrm{N}=27)$, spices $(\mathrm{N}=13)$, and fruits $(\mathrm{N}=12)$. In case of plants, fruit was the main part used, followed by seed, and leaf, similarly in animals, flesh was the most often used part. The maximum number of species reported from an ethnic group was by Kashmiri, while the Gujjar reported the minimum number of species used. During cultural usage, festivals and marriages showed greater similarity of species usage, whereas the least overlap was observed between funerals and marriages. In case of cultural recipes, festivals and marriages showed greater similarity, whereas the least overlap was observed between festivals and funerals.

Conclusion. According to the findings of the current study, all ethnic groups living the Kashmir valley have a profound cultural link to the flora and fauna, particularly when it comes to food. This is the first complete research 
of the flora and fauna utilized in diverse dishes in different ethnic groups' cultural events, and it will aid in the preservation of traditional gastronomic expertise in Kashmir's valley.

Keywords: Recipes; Cultural occasions; Ethnic groups; Marriage; Funeral; Festivals

\section{Background}

The Himalayan region occupies a distinct place in the Indian subcontinent as it also harbors a unique cultural diversity (Wadhawan 2021). The predominantly Himalayan Kashmir valley (Vale of Kashmir) is famous for its serene beauty and cultural richness (Khanna and Bhagat 2021). The culture of Kashmir valley in India is very diverse, multicolored, and multifaceted, it is a brilliant blend of different ethnic groups and social entities, result of the merger of numerous civilizations that have inhabited the valley during time and left their imprint on the local culture (Rather et al.2016). Kashmir is said to have close cultural relations with the present-day China, Tibet, Central Asian cities, Afghanistan, and Iran (Sheikh and Shabina 2011).

Culture has been described as the customary beliefs, social forms, and material traits of a racial, religious, or social group; the characteristic features of everyday existence shared by people in place or time since the $19^{\text {th }}$ century (Tey et al. 2018). Ethnic foods evolved in human culture, and the phenomena of human biology have significant socio-cultural values (Phasha et al. 2020). In India diverse communities have developed unique food patterns. Punjabis are e.g, known for Paharata, Biharis for Littichoka, and in south India Idli, samabar, and dosa are very common foods (Reardon and Minten 2011). Kashmir is characterized by unique food recipes, languages, dress, customs, rituals, festivals, and communities. As a clear expression of cultural values, food becomes a central identity marker, defining personality, social class, lifestyles, and relationships, from family, to community, to ethnic groups or nationality, changing through time and place (Boutaud et al. 2016). In Certain foods are highly prized; others are reserved for religious feasts, still others are a mark of social position (Reddy and Anitha 2015). Food permeates each and every aspect of human beings. As sociologist Claude Fischler notes, "Food not only nourishes but also signifies" (Fischler 1988).

In Kashmir different ethnic communities (Kashmiri, Pahari, and Gujjar) are unique in their cultural expressions (Snedden 2021). All these communities use different plants and animals for their cultural food dishes. Rice, the staple food in Kashmir, used from ancient times (Kaw 2010), and is used in almost all communities, and in Kashmiri known as "Baath". Some famous local dishes include Haakh, Razma-daal which are obtained from collard green and beans respectively. Locally it is said that the food should both taste and look good and its aroma must be appetizing. Success of a meal lies in its appeal to the eyes, nose and then the tongue. In Kashmir, cultural occasions like marriages, parties, and festivals (Eid, Eid-e-milad, Holi, Diwali), are celebrated by consuming a wide variety of dishes. In addition to the sedifferent food dishes, there are several other dishes served during funerals. Spices and condiments, commonly called 'Masaale', play a very important role in the Kashmiri Culinary Art and Cuisine, especially in the dishes of 'wazwaan'. Meanwhile different people from the valley speak about the ethno-food through different veils, some calling it legacy, and some saying that the majority of ethno-foods are only for the elite class, while some see it as an undue evolution of traditional food.

Because Kashmir has rich ethnic food and cultural diversity, the present study focused on the comprehensive assessment regarding the usage of plants, mammals, fish, and birds in ethnic foods with following objectives; (i) to have an in-depth understanding of relation between food and culture in the valley (Kashmir), (2) to study the crosscultural usage of species in specific occasions and principal recipes (3) to study the variation in the number of recipes associated with the cultural occasions across the Kashmir valley. By answering these questions, we provide fundamental information about the gastronomic use of species (plants, mammals, fish, and birds) in the valley, which can serve as a benchmark for the preservation of traditional gastronomic knowledge in the valley (Kashmir).

\section{Materials and Methods}

\section{Study area}

The valley of Kashmir is located between the Karakoram and the Pir-Panjal ranges, is bordered by the Jammu division to the south, Ladakh division to the east, and the Line of Control with Pakistan in the north and the west (Hassan et al. 2021) and lies between $33^{\circ} 22^{\prime}$ to $34^{\circ} 0^{\prime} \mathrm{N}$ and $73^{\circ} 55^{\prime}$ to $73^{\circ} 33^{\prime} \mathrm{E}$ (Fig.1). The Valley spreads over an area of $15948 \mathrm{~km}^{2}$ divided into ten administrative districts with an altitude range from 550 to 5000 masl (Haq et al. 2020). More than 90 percent of the population are Muslim, and rest are Hindu and Sikhs ( $\mathrm{ECl} 2018)$. The climate is temperate continental-Mediterranean. Maximum rainfall occurs in March-April, with some heavy showers during July and August on account of the Southwest Indian Monsoon (Gairola et al. 2014). The area is surrounded by the 
high Pir-Panjal range to the south and the high Zanskar range to the north, protecting the valley from high monsoon rainfall and cold blasts from Ladakh and Tibet. Kashmiri and Urdu are the main languages spoken. Other languages include Paharii, and Gojrii. Kashmiri, Dogri, Hindi. Ethnic groups are Gujjar, Kashmiri, Pahari (Hassan et al. 2021; Asif et al. 2021). All communities of the valley are both vegetarian and non-vegetarian. Spices have a unique place in the cultural recipes grown locally and some are imported from other states. Along with beautiful landscape, cultural food stuffs like apple, saffron, almond, and walnut have national as well as international fame (Rather et al. 2016).

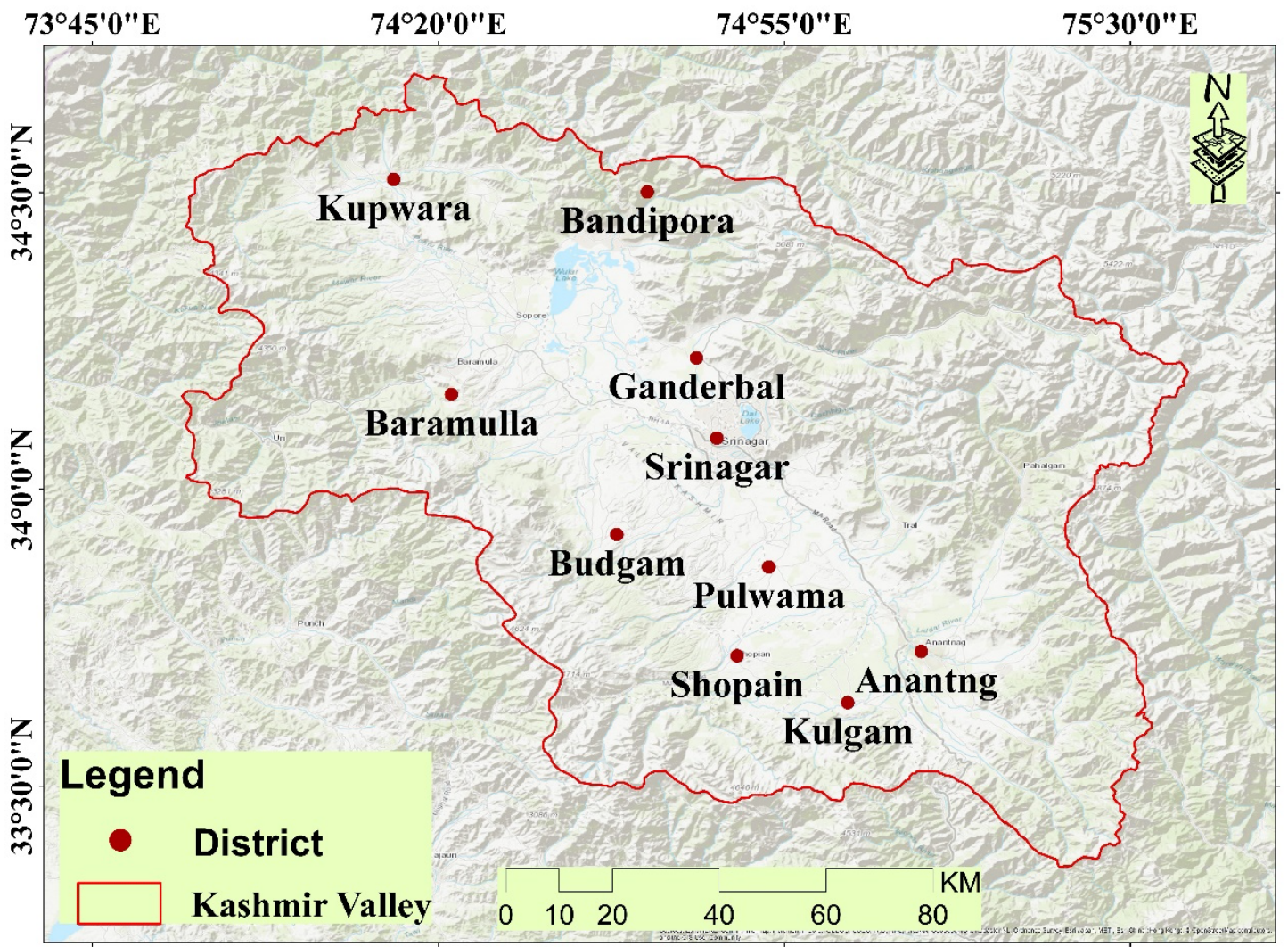

Figure 1. Map of the Kashmir valley and points showing location of districts in the study area. GIS Software: ArcGIS (Esri) 10.1.

\section{Materials and Methods}

The present study was based on a carefully planned field survey carried out in all ten administrative districts of Kashmir valley. The data was collected in 2020-2021through face-to-face interviews using semi-structured questionnaires (Hassan et al. 2021; Haq et al. 2020; Mir et al., 2021). The informants include different groups like Waza (chef), housewives, street food vendors, restaurant owners, and local people. The most important knowledge holder group was the Wazas. The interviewed people were asked: whether the species (plants, mammals, birds, and fish) were used in their cultural occasions, name of the food recipes obtained from the particular species, recipes used in cultural occasions like festivals, marriage, and funerals, the part of the species used in the recipe and principal species used in special recipes (Fig. 2).

\section{Data analysis}

To determine the relationship between various recipes and species a Two-way cluster analysis was conducted using PAST software ver.3.14. (Asif et al. 2021). The presence/absence (1/0) data was subjected to the formation of hierarchical agglomerative clusters, to elucidate similarities and differences at species and recipes level. Principal components analysis (PCA) was used to find hypothetical variables (components) that accounted for as much of the variance in our multidimensional data as possible (Haq et al. 2020a; Haq et al. 2021). A Venn diagram was created using Bioinformatics \& Evolutionary Genomics software (Altaf et al. 2021) (https://bioinformatics.psb.ugent.be/webtools/Venn/) 


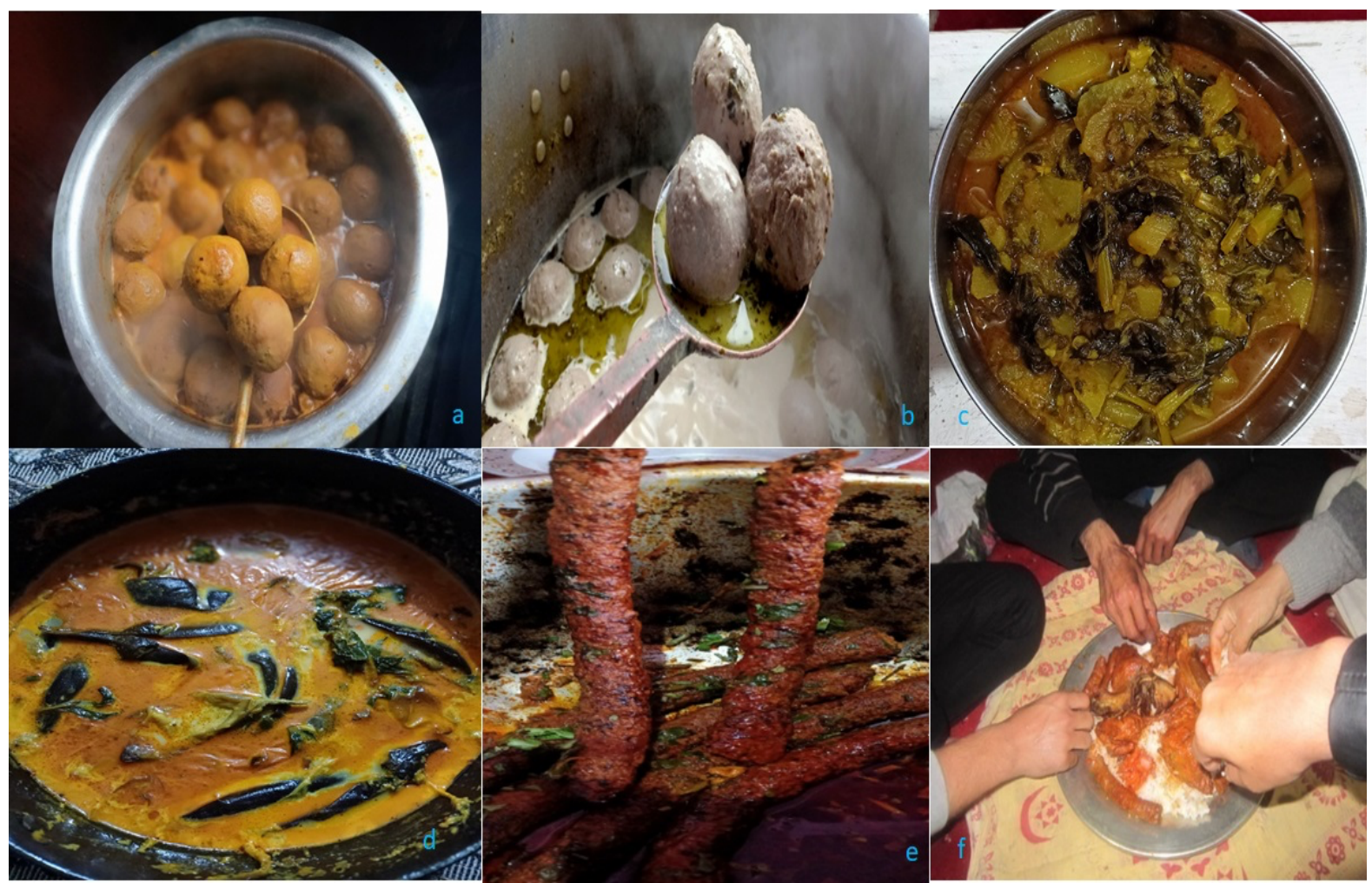

Figure 2. Photographs of some important dishes. (a) Rista, (b)Gushtaba, (C) Haak, (d) Wangan, (e) Kabab (f) Traim (feast for four people together) offered to guests at marriage ceremonies.

\section{Results}

\section{Demographic details of respondents and their views about cultural food and food species}

We conducted interviews with 298 informants, of which 117 were from North Kashmir, 94 from Central Kashmir, and 87 from South Kashmir. Three ethnic groups were selected i.e., Kashmiri (38.59\%), Pahari (31.87\%), and Gujjar (29.53\%). The selected ethnic groups are the main ethnic groups of the valley (Hassan et al. 2021). During the survey respondents showed an even gender distribution, where 151 were men and 146 were women. The age of the respondents ranged from 20 years to 55 years. The respondents interviewed included wazas [Chef] (27\%), housewives (21\%), street food vendors (18\%), restaurant owners (17\%), and other local people (18\%). Most of the respondents belonged to age 35-50 (187). More than half of the respondents had not received any formal education (57.04\%), others had merely primary education (35.23\%) followed by $(7.71 \%)$ having education up to $10^{\text {th }}$ standard (Table.1).

A large portion (78\%) treated cultural food as the heritage of the valley (Kashmir). In contrast (13\%) believed it as elite class food, while $9 \%$ thought it was non-essential and society made its evolution with time causing a burden to the economically weaker section.

Table 1. Demographic status of the respondents from the study area.

\begin{tabular}{|l|l|l|}
\hline DEMOGRAPHIC FEATURES & NUMBER OF PEOPLE & PERCENTAGE \\
\hline Bio-geographic areas & & \\
\hline North Kashmir & 117 & 39 \\
\hline South Kashmir & 87 & 29 \\
\hline Central Kashmir & 94 & 32 \\
\hline Ethnic communities & & \\
\hline Gujjar & 63 & 21 \\
\hline Pahari & 72 & 24 \\
\hline Kashmiri & 163 & 54 \\
\hline Education & 170 & \\
\hline Illiterate & 105 & 57. \\
\hline Primary education & 23 & 35. \\
\hline Secondary education & & 8 \\
\hline
\end{tabular}




\begin{tabular}{|l|l|l|}
\hline Age range (Years) & \multicolumn{2}{l|}{} \\
\hline $25-35$ & 26 & 9 \\
\hline $35-50$ & 187 & 63 \\
\hline $50-60$ & 85 & 28 \\
\hline Ethnic Groups & \multicolumn{2}{|l|}{} \\
\hline Kashmiri & 115 & 39 \\
\hline Pahari & 95 & 32 \\
\hline Gujjar & 88 & 30 \\
\hline Professional groups & & \\
\hline Wazas & 82 & 27 \\
\hline Housewives & 63 & 21 \\
\hline Street food vendors & 54 & 18 \\
\hline Restaurant owners & 51 & 17 \\
\hline Local people & 48 & 16 \\
\hline Gender & & \\
\hline Male & 152 & 51 \\
\hline Female & 146 & 49 \\
\hline
\end{tabular}

\section{Documentation of food species}

The present study recorded75edible species used in cultural foods in the valley (Kashmir) (Table 2). All the documented species belonged to 32 families. The dominant families were Brassicaceae (8\%) and Fabaceae (8\%) in vegetables, spices, and fruits, while in fish and mammals Cyprinidae (6\%) and Bovidae (6\%) were the leading families (Fig. 3).

Our documentation revealed that mammals included 6 species out of which 5 belonged to Bovidae and the sixth one species belonged to Camelidae. Fish were found to have 5 species, all from Cyprinidae. In case of birds, 12 species were recorded, among which 4 species belonged to Anatidae, 1 to Ardeidae, 3 to Columbidae, 2 to Phasianidae, and 1 to Picidae. A total of 27 species of vegetables were recorded in ethnofood usage, among which maximum ( $\mathrm{N}=6)$ species belonged to family Brassicaceae and Fabaceae each. Fruits were recorded with 12 different species, Rosaceae $(\mathrm{N}=2)$, Bromeliaceae $(\mathrm{N}=1)$, Rutaceae $(\mathrm{N}=2)$, Solanaceae $(\mathrm{N}=2)$, Cucurbitaceae $(\mathrm{N}=2)$, Lythraceae $(N=1)$, Juglandaceae $(N=1)$, Anacardiaceae $(N=1)$, and "Malvaceae" $(N=1)$. Thirteen plants were documented as spices, families included Lauraceae $(\mathrm{N}=2)$, Zingiberaceae $(\mathrm{N}=4)$, Piperaceae $(\mathrm{N}=1)$, Myrtaceae $(\mathrm{N}=1)$, Ranunculaceae $(\mathrm{N}=1)$, Lamiaceae $(\mathrm{N}=1)$, Solanaceae $(\mathrm{N}=1)$, Iridaceae $(\mathrm{N}=1)$, Amaranthaceae $(\mathrm{N}=1)$.

\section{Cross cultural usage}

The maximum unique number of species were reported by the Kashmiri ethnic group (Fig. 4), while the Gujjar reported no such species. The Kashmiri and Pahari showed greater similarity, whereas least overlap was observed between Kashmiri and Gujjar. Cross cultural comparison of species showed that 45 species were commonly used by all ethnic groups

\section{Cultural usage of species and principal recipes}

The maximum usage of species was reported at festivals occasion, while the marriage occasion reported minimum usage of species. (Fig. 5a). The festivals and marriages showed greater similarity, whereas the least overlap was observed between funerals and marriages. A cross-cultural comparison of resources showed that 30 species were overlapping between the three cultural occasions of the study area.

Across the different cultural occasions, recipes showed significant variation $\left(\chi^{2}=22.42, d f=27, p<0.024\right)$. A total of 10 recipes (containing Spinacia oleracea, Brassica oleracea var. viridis, Nelumbo nucifera, Brassica rapa var. rapa, Cajanus cajan, Solanum lycopersicum, Raphanus raphanistrum, Cicer arietinum, Vigna radiata, Phaseolus vulgaris) were unique to funerals, 6 recipes (Tang, Methi-maaz, Pulao, kantii, Marchavagun-kurma, Tabakh-maaz, Rista, Aabgosh, Yakhin) unique to marriages and 4 recipes (Tailth-gad, Shabdaig, Tikken, Muj-Gad) were unique to festivals. Common recipes for festivals, funerals and marriages included Rogan-josh, Baath, Yakhin, Salaad. For festivals and marriages common recipes ( $\mathrm{N}=4$ ) were Gushtab, Kabab, Nadir-gad, Rista, and common recipes for festivals and funerals were Nadi and Aloo chips. There was one recipe shared between funerals and marriages (Methi-maaz) Festivals and marriage showed greater similarity, whereas the least overlap was observed between festivals and 
funerals (Fig. 5b). The usage of different recipes by the various ethnic groups (Gujjar, Kashmiri, Pahari) can be observed in Table. 2.

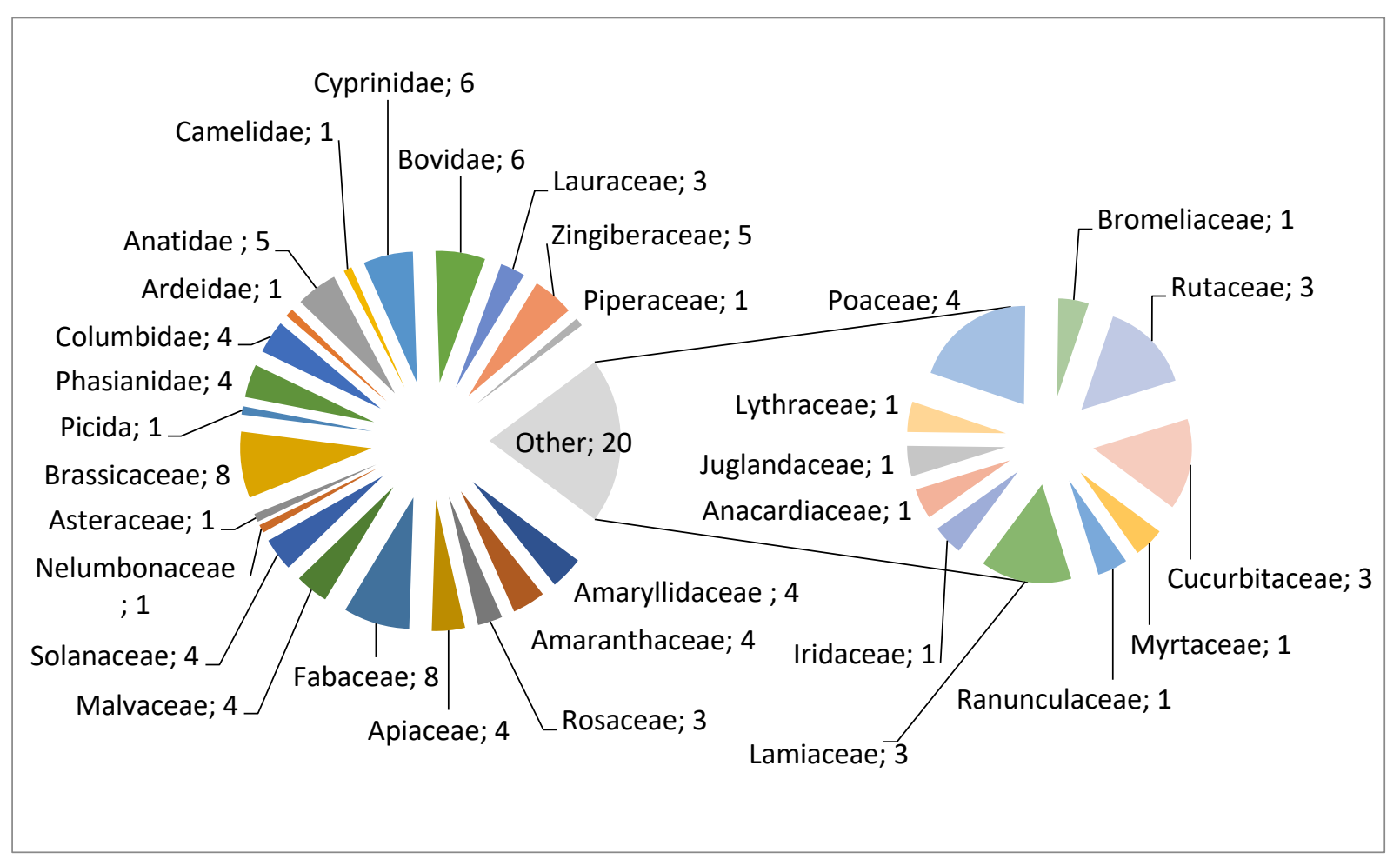

Figure 3. Percentage of species (family) used in ethno-foods of Kashmir valley

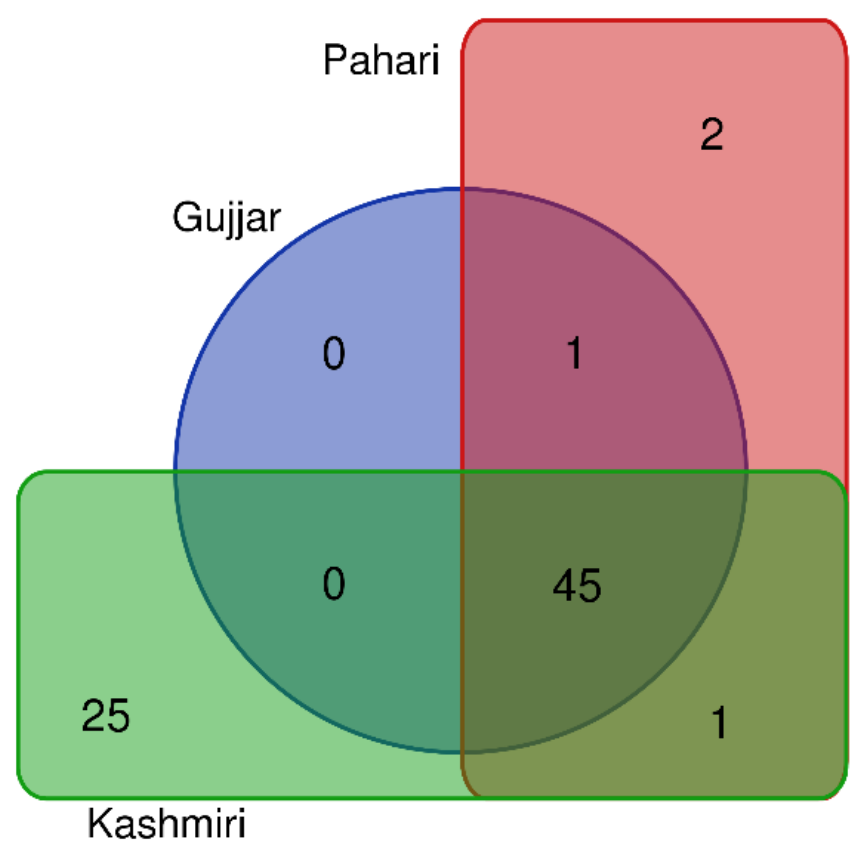

Figure 4. Unique and common species across ethnic groups (Gujjar, Pahari, Kashmiri)

\section{Parts of ethno-foods used in cultural occasions}

We found that different parts of both plants and mammals were used in different recipes. In case of mammals, flesh (24\%) was the most prime part used followed by legs (19\%), liver (19\%), heart (19\%), lungs (7\%) (Fig. 6a). In case of plants, fruits (30\%) were the main parts used followed by seed (24\%), leaf (24\%), stem (7\%), root (7\%), flower (6\%) and bark (2\%) (Fig. 6b). 


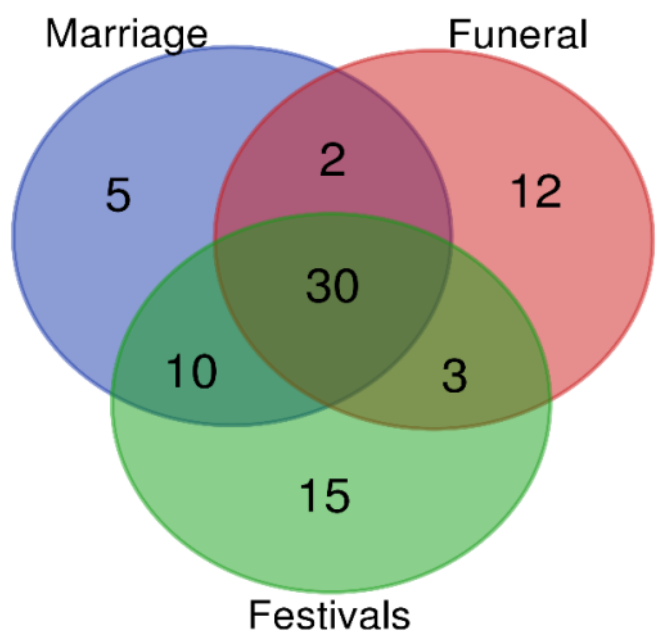

a

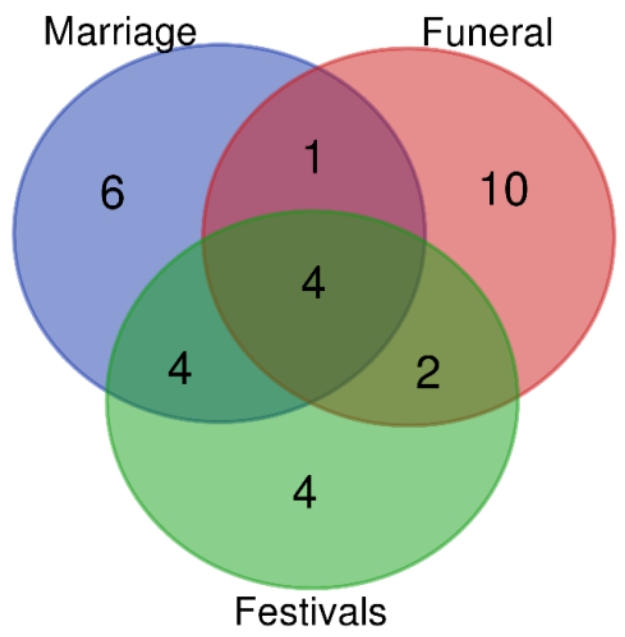

b

Figure 5. Venn diagram (a) representing unique and common species on cultural occasions (Marriage, Festivals, Funeral) (b) representing unique and common recipes on cultural occasions (Marriage, Festivals, Funeral)

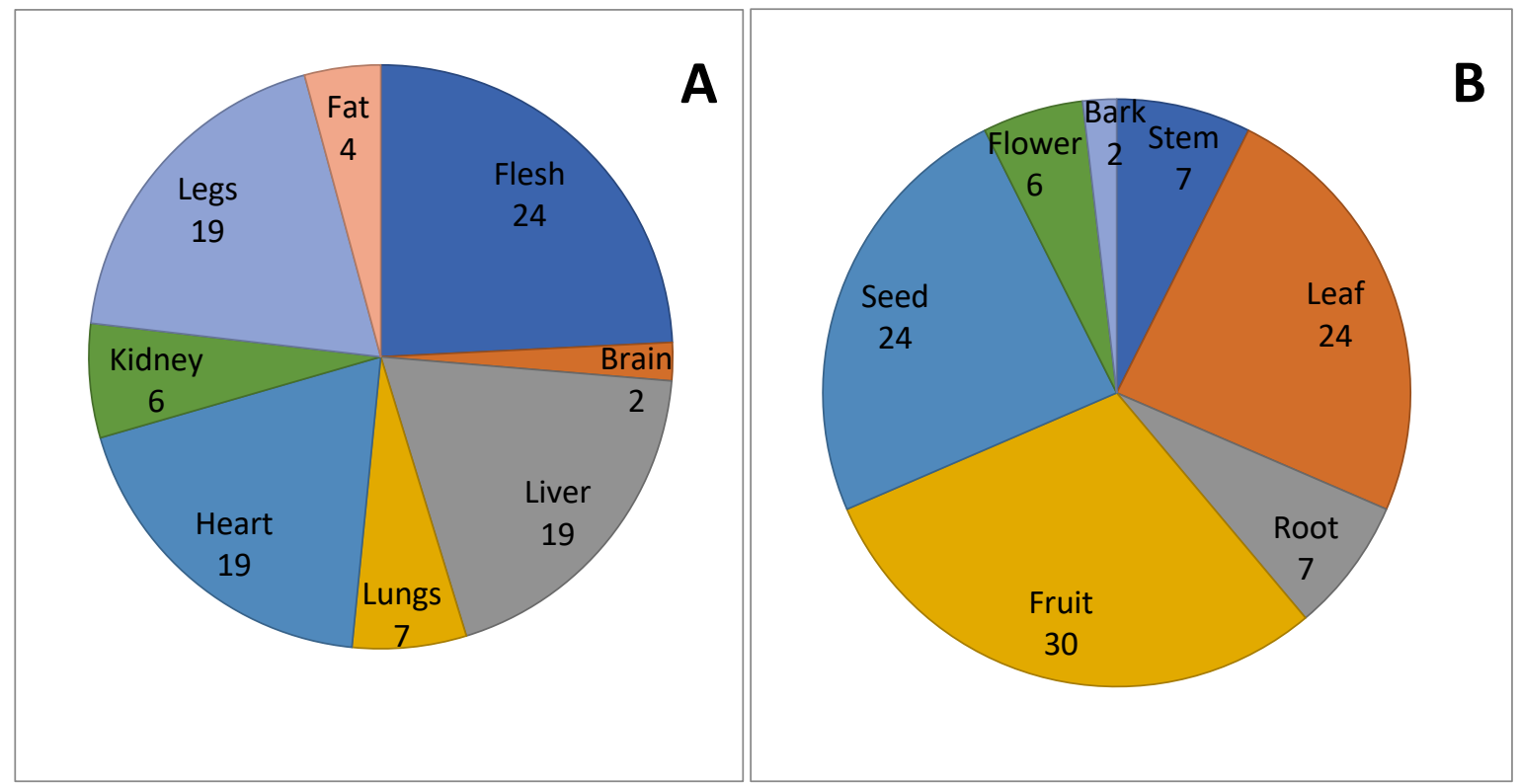

Figure 6. Percentage of different parts of (a) animal; (b) plant species used in ethno-foods

\section{Classification of species concerned with respect to the principal recipes}

The Two-Way Cluster Analyses of 28 ethno-food recipes included 47 food species resulting in 2 major clusters (Fig. 7) recognized by the Sorenson's similarity index. Mammal species grouped in their respective limbs in the cluster have more similar usage and show proximity to each other. Group one included 13 species and the corresponding recipes were, Tailth Gad, Tabakh-maaz, Haak, Paalak, Muj, Baath, Gaguj and Salaad. Group two included 34 species and the corresponding recipes were Kabab,Rista, Kantii, Muj-Gad, Nadir-Gad, Shabdaig, Tamatar, Razma-daal, Masour-daal, Muang-daal, Nadir, Rogan-josh, Marchavagun-Kurma, Gushtab, Yakhin, Aab-gosh (Fig. 7).

Similarly, the PCA showed considerable variation in different recipes, in which specific groups of species were more related to a particular recipe than others (Fig.8). For example, PC1 and PC2 explained species distribution in the biplot, in which species grouped into separate cluster based on species presence/absence can be identified in Fig. 8. 


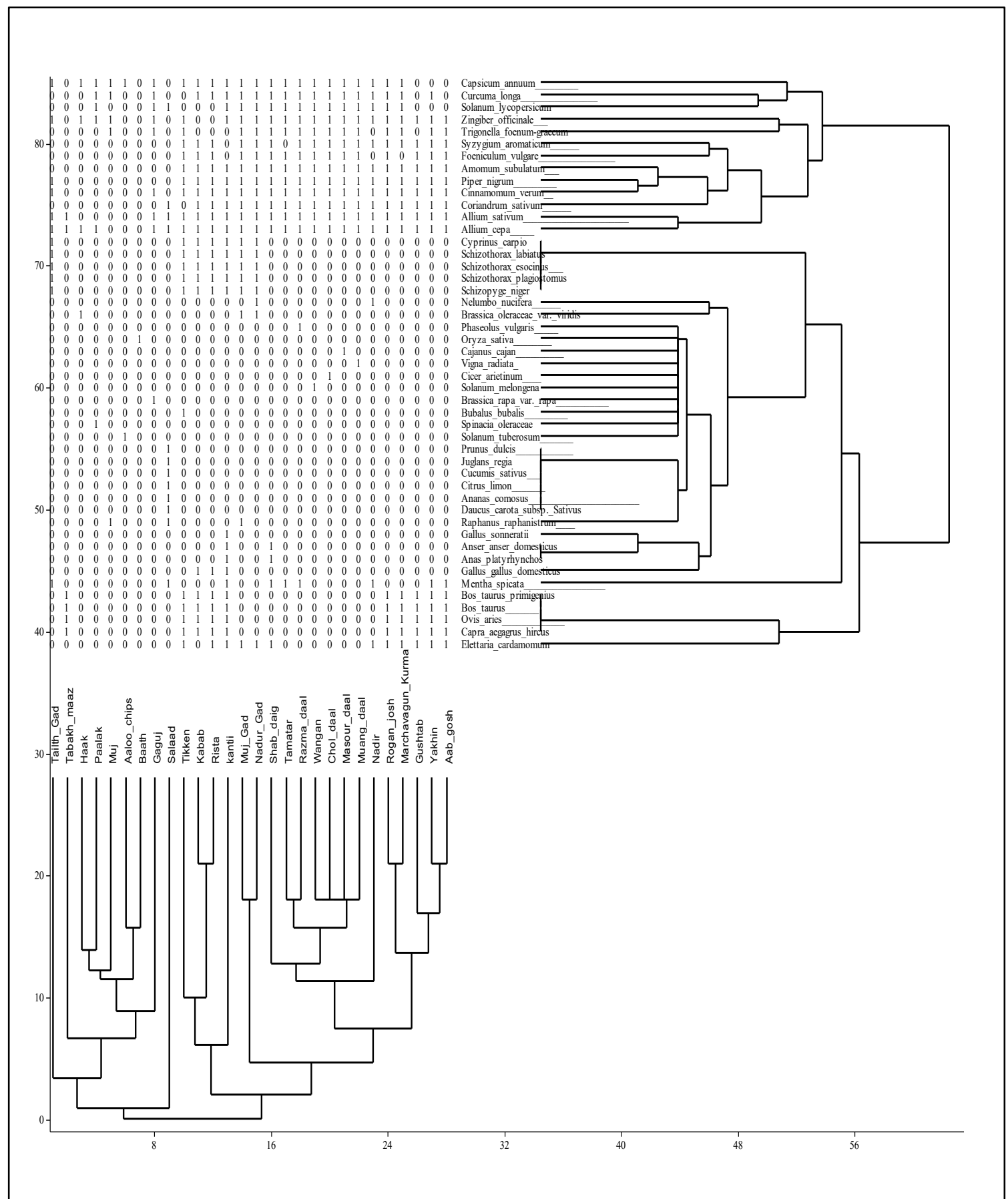

Figure 7. Two Way Cluster Analysis based on Sorenson's similarity index of species and recipes. *The presence of the species in the figure is represented by 1 and absence by 0 .

\section{Variation in the usage pattern of recipes across the valley}

As Srinagar is the summer capital of the UT (Jammu and Kashmir) and is the only city in the Kashmir valley, it is the prime region connected with the rest of the country and the world. The basis of the evolution of culture in the valley finds its roots in this city. In case of recipes associated with the cultural occasions Srinagar was found at the second position (27), followed by Pulwama (26), Shopian (25) and others. The district with maximum number of recipes used in cultural occasion was Anantnag (28) and the district with least number of recipes in ethno-cultural occasions was Kupwara (22)(Fig.9). 


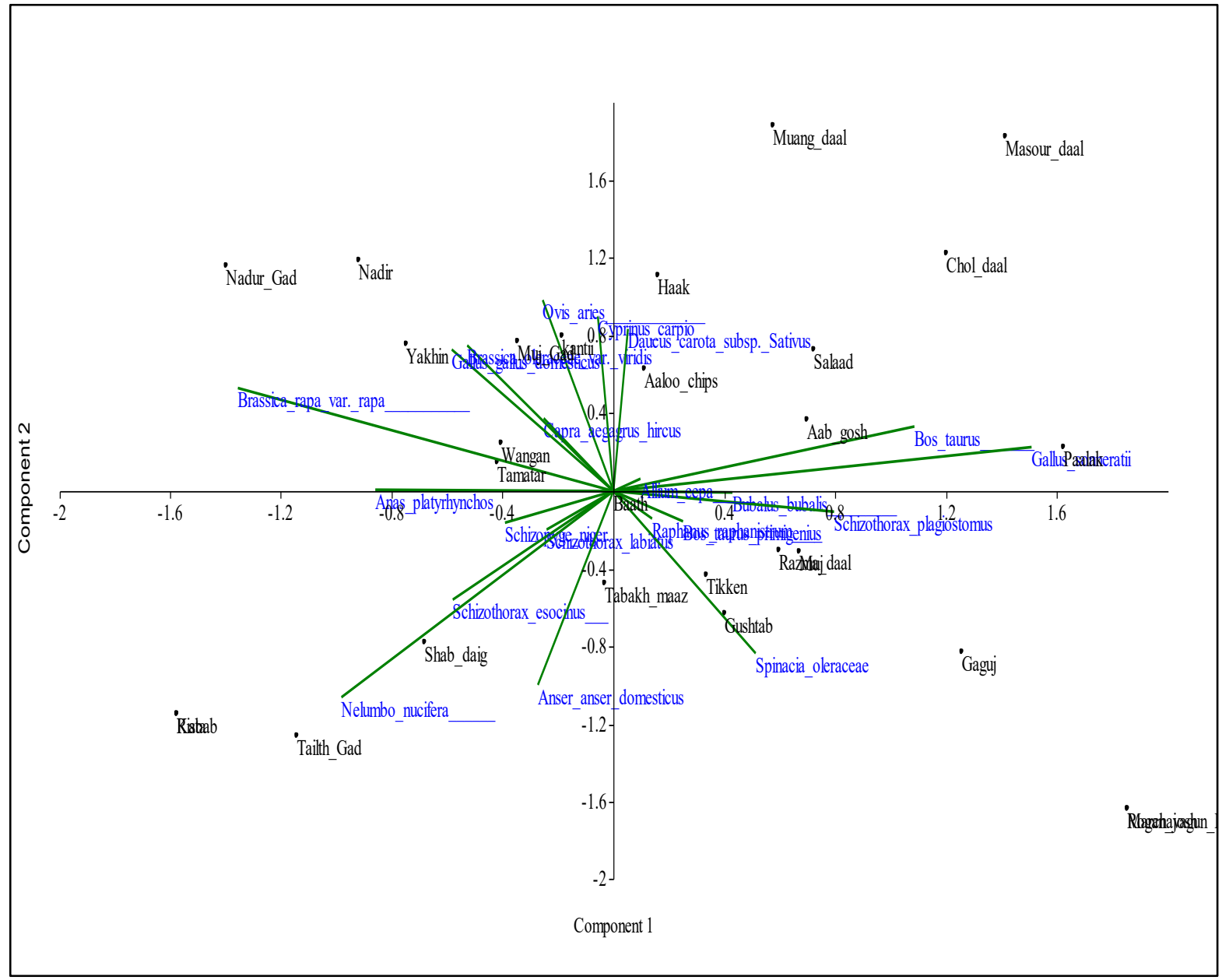

Figure 8. Principal Component Analyses (PCA) biplot of different recipes and food in Kashmir Himalaya.

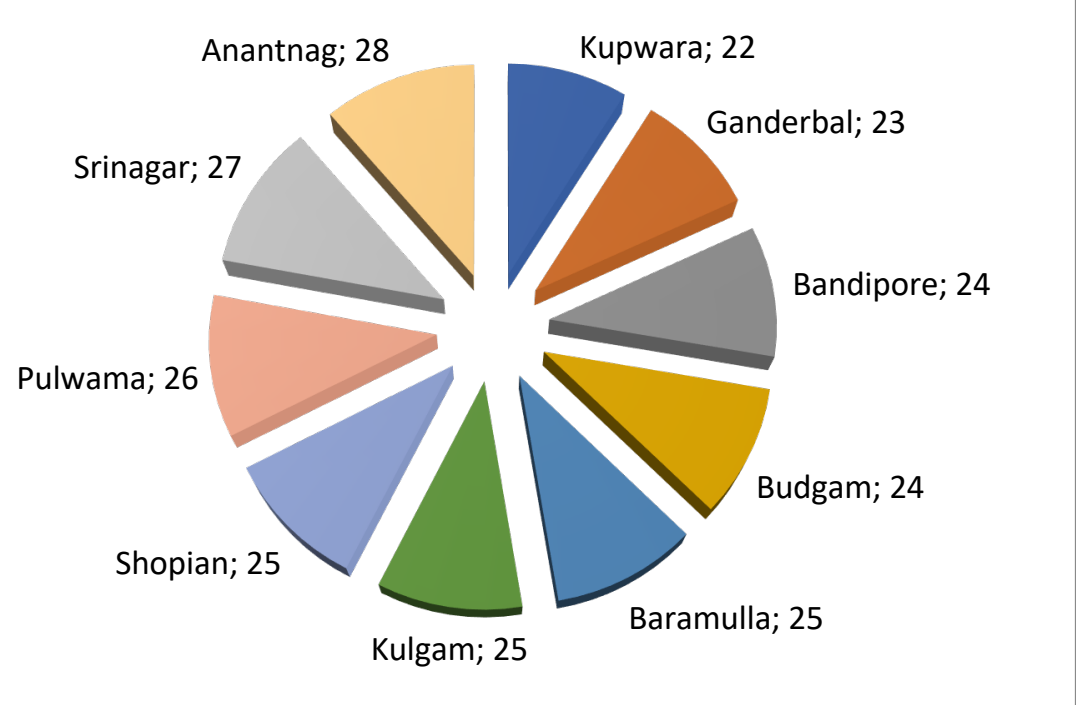

Figure 9. Number of recipes used in all administrative districts of Kashmir 


\section{Ethnobotany Research and Applications}

Table 2. Inventory of species used in ethno-foods from the valley (Kashmir)

\begin{tabular}{|c|c|c|c|c|c|}
\hline $\begin{array}{l}\text { ANIMALS } \\
\text { Scientific Name } \\
\text { (Family) }\end{array}$ & English Name & Local Name & $\begin{array}{l}\text { Part } \\
\text { Used }\end{array}$ & Recipes & Ethnic Groups \\
\hline $\begin{array}{l}\text { Anas acuta (Linnaeus, 1758) } \\
\text { (Anatidae) }\end{array}$ & Northern pintail & Pechin & $\begin{array}{l}\text { Flesh } \\
\text { Liver } \\
\text { Heart } \\
\text { Legs }\end{array}$ & Shakaar-maaz & Kashmiri \\
\hline $\begin{array}{l}\text { Anas platyrhynchos } \\
\text { (Anatidae) }\end{array}$ & Duck & Bataich & $\begin{array}{l}\text { Flesh } \\
\text { Liver } \\
\text { Heart } \\
\text { Legs }\end{array}$ & Shabdaig, Kantii & Kashmiri \\
\hline $\begin{array}{l}\text { Anseranser domesticus } \\
\text { (Linnaeus, 1758) } \\
\text { (Anatidae) }\end{array}$ & Goose & Anz & $\begin{array}{l}\text { Flesh } \\
\text { Liver } \\
\text { Heart } \\
\text { Legs }\end{array}$ & $\begin{array}{l}\text { Shabdaig, Kantii } \\
\text { *Shabdaig is prepared by cooking duck or goose } \\
\text { with pumpkin. }\end{array}$ & Kashmiri \\
\hline $\begin{array}{l}\text { Bos taurus (Linnaeus, 1758) } \\
\text { (Bovidae) }\end{array}$ & Cow & $\begin{array}{l}\text { Gaav } \\
\text { Gaan }\end{array}$ & $\begin{array}{l}\text { Flesh } \\
\text { Liver } \\
\text { Lungs } \\
\text { Heart } \\
\text { Kidney } \\
\text { Legs } \\
\text { Fat }\end{array}$ & $\begin{array}{l}\text { Rista, Kabab, Gushtab, Rogan-josh, Marchavagun- } \\
\text { kurma, Tabakh-maaz, Yakhin, Aab-gosh, Tikken, } \\
\text { Kantii, Methi-maaz, Tabakh-maaz } \\
\text { *Rogan-josh is made simply by cooking the meat } \\
\text { with oil and `spices. } \\
\text { *Marchavagun-kurma is made similarly as Rogan- } \\
\text { josh by adding a lot of paprika. } \\
\text { *Tabakh-maaz, is obtained by cutting ribs into } \\
\text { pieces then fried. }\end{array}$ & $\begin{array}{l}\text { Kashmiri } \\
\text { Gujjar } \\
\text { Pahari }\end{array}$ \\
\hline $\begin{array}{l}\text { Bos taurus primigenius } \\
\text { (Linnaeus, 1758) } \\
\text { (Bovidae) }\end{array}$ & $O x$ & Daand & $\begin{array}{l}\text { Flesh } \\
\text { Liver } \\
\text { Lungs } \\
\text { Heart } \\
\text { Kidney } \\
\text { Legs }\end{array}$ & $\begin{array}{l}\text { Rista, Kabab, Gushtab, Rogan-josh, Marchavagun- } \\
\text { kurma, Tabakh-maaz, Yakhin, Aab-gosh, Tikken, } \\
\text { Kantii, Methi-maaz, Tabakh-maaz } \\
\text { *Rista and Gustab are meat balls made by } \\
\text { grinding meat on a traditional stone and then } \\
\text { cooked. } \\
\text { *Kabab is made by chopping the meat with knife } \\
\text { and then spreaded over a rod and finally roasted. }\end{array}$ & $\begin{array}{l}\text { Kashmiri } \\
\text { Gujjar } \\
\text { Pahari }\end{array}$ \\
\hline $\begin{array}{l}\text { Bubalus bubalis (Linnaeus, } \\
\text { 1758) } \\
\text { (Bovidae) }\end{array}$ & Buffalo & $\begin{array}{l}\text { Moonsh } \\
\text { Mains }\end{array}$ & $\begin{array}{l}\text { Flesh } \\
\text { Liver } \\
\text { Lungs } \\
\text { Heart } \\
\text { Kidney }\end{array}$ & $\begin{array}{l}\text { Tikken } \\
\text { *Tikken are made by chopping the meat, making } \\
\text { into pads and then fried. }\end{array}$ & $\begin{array}{l}\text { Gujjar } \\
\text { Pahari }\end{array}$ \\
\hline
\end{tabular}


Ethnobotany Research and Applications

\begin{tabular}{|c|c|c|c|c|c|}
\hline & & & Legs & & \\
\hline $\begin{array}{l}\text { Camelus dromedaries } \\
\text { (Linnaeus, 1758) } \\
\text { (Camelidae) }\end{array}$ & Camel & Oount & $\begin{array}{l}\text { Flesh } \\
\text { Liver } \\
\text { Lungs } \\
\text { Heart } \\
\text { Kidney } \\
\text { Legs } \\
\text { Fat }\end{array}$ & $\begin{array}{l}\text { Rista, Kabab, Gushtab, Rogan-josh, Marchavagun- } \\
\text { kurma, Tabakh-maaz, Yakhin, Aab gosh, Tikken, } \\
\text { Kantii }\end{array}$ & Pahari \\
\hline $\begin{array}{l}\text { Capra aegagrushircus } \\
\text { (Linnaeus, 1758) } \\
\text { (Bovidae) }\end{array}$ & Goat & $\begin{array}{l}\text { Chaavaj } \\
\text { Kat } \\
\text { Bakrie } \\
\text { Bkra }\end{array}$ & $\begin{array}{l}\text { Flesh } \\
\text { Liver } \\
\text { Lungs } \\
\text { Heart } \\
\text { Kidney } \\
\text { Legs } \\
\text { Fat } \\
\text { Brain }\end{array}$ & $\begin{array}{l}\text { Rista, Kabab, Gushtab, Rogan-josh, Marchavagun- } \\
\text { kurma, Tabakhmaaz, Yakhin, Aab gosh, Tikken, } \\
\text { kantii, methi-maaz } \\
\text { *Kantii is prepared by cutttig meat into small } \\
\text { pieces then fried with onion. } \\
\text { *Methi-maaz is prepared by cooking meat with } \\
\text { fenugreek leaves. }\end{array}$ & $\begin{array}{l}\text { Kashmiri } \\
\text { Gujjar } \\
\text { Pahari }\end{array}$ \\
\hline $\begin{array}{l}\text { Columba livia (Gmelin, } \\
\text { 1789) } \\
\text { (Columbidae) }\end{array}$ & Rock Pigeon & $\begin{array}{l}\text { Kotur } \\
\text { Kabotur }\end{array}$ & $\begin{array}{l}\text { Flesh } \\
\text { Liver } \\
\text { Heart } \\
\text { Legs }\end{array}$ & $\begin{array}{l}\text { Kotar-maaz } \\
\text { *Kotar-maaz is prepared by cooking or frying the } \\
\text { meat of pigeon. }\end{array}$ & $\begin{array}{l}\text { Kashmiri } \\
\text { Gujjar } \\
\text { Pahari }\end{array}$ \\
\hline $\begin{array}{l}\text { Cyprinus carpio (Linnaeus, } \\
1758) \\
\text { (Cyprinidae) }\end{array}$ & Common carp & Punjabe Gad & Flesh & $\begin{array}{l}\text { Rista, Kabab, Muj-gad, Nadur-gad, Tikken, Tailth- } \\
\text { gad, Kantii }\end{array}$ & Kashmiri \\
\hline $\begin{array}{l}\text { Gallus gallus domesticus } \\
\text { (Linnaeus, 1758) } \\
\text { (Phasdanidae) }\end{array}$ & Broiler (Chicken) & Boiler & $\begin{array}{l}\text { Flesh } \\
\text { Liver } \\
\text { Heart } \\
\text { Legs }\end{array}$ & Rista, Kabab, Kantii & $\begin{array}{l}\text { Kashmiri } \\
\text { Gujjar } \\
\text { Pahari }\end{array}$ \\
\hline $\begin{array}{l}\text { Gallus sonneratii } \\
\text { (Temminck, 1813) } \\
\text { (Phasianidae) }\end{array}$ & Kashmiri jungle fowl & $\begin{array}{l}\text { Van Kukur } \\
\text { JangliKukud }\end{array}$ & $\begin{array}{l}\text { Flesh } \\
\text { Liver } \\
\text { Heart } \\
\text { Legs }\end{array}$ & Kantii & Pahari \\
\hline $\begin{array}{l}\text { Ixobrychus minutus } \\
\text { (Linnaeus, 1766) } \\
\text { (Ardeidae) }\end{array}$ & Little bittern & Gui & $\begin{array}{l}\text { Flesh } \\
\text { Liver } \\
\text { Heart } \\
\text { Legs }\end{array}$ & Shakaar-maaz & Kashmiri \\
\hline $\begin{array}{l}\text { Jynx torquilla (Linnaeus, } \\
1758) \\
\text { (Picidae) }\end{array}$ & Eurasian wryneck & Viri Mot & $\begin{array}{l}\text { Flesh } \\
\text { Liver } \\
\text { Heart } \\
\text { Legs }\end{array}$ & Shakaar-maaz & Kashmiri \\
\hline $\begin{array}{l}\text { Lophophorus impejanus } \\
\text { (Latham, 1790) }\end{array}$ & Himalayan Monal & $\begin{array}{l}\text { Wan Kokur } \\
\text { Son Murg }\end{array}$ & $\begin{array}{l}\text { Flesh } \\
\text { Liver }\end{array}$ & Shakaar-maaz & $\begin{array}{l}\text { Kashmiri } \\
\text { Gujjar }\end{array}$ \\
\hline
\end{tabular}




\section{Ethnobotany Research and Applications}

\begin{tabular}{|c|c|c|c|c|c|}
\hline (Phasianidae) & & & $\begin{array}{l}\text { Heart } \\
\text { Legs }\end{array}$ & & Pahari \\
\hline $\begin{array}{l}\text { Ovis aries (Linnaeus, 1758) } \\
\text { (Bovidae) }\end{array}$ & Sheep & $\begin{array}{l}\text { Kath } \\
\text { Laayla } \\
\text { Payhaad }\end{array}$ & $\begin{array}{l}\text { Flesh } \\
\text { Liver } \\
\text { Lungs } \\
\text { Heart } \\
\text { Kidney } \\
\text { Legs } \\
\text { Fat } \\
\text { Brain }\end{array}$ & $\begin{array}{l}\text { Rista, Kabab, Gushtab, Rogan-josh, Marchavagun- } \\
\text { kurma, Tabakh-maaz, Yakhin, Aab gosh, Tikken, } \\
\text { Kantii,Methi-maaz, Tabakh-maaz } \\
\text { *Aab gosh are made by cooking meat in milk } \\
\text { with small quantity of sugar. } \\
\text { *Yakhin is prepared by boiling curd with butter } \\
\text { and spices. }\end{array}$ & $\begin{array}{l}\text { Kashmiri } \\
\text { Gujjar } \\
\text { Pahari }\end{array}$ \\
\hline $\begin{array}{l}\text { Schizo pygeniger (Heckel, } \\
\text { 1838) } \\
\text { (Cyprinidae) }\end{array}$ & Alghad Snow trout & Ale Gad & Flesh & $\begin{array}{l}\text { Rista, Kabab, Muj-gad, Nadur-gad, Tikken, Tailth- } \\
\text { gad, Kantii } \\
\text { *Muj- gad is prepared by cooking fish with } \\
\text { Radish. }\end{array}$ & Kashmiri \\
\hline $\begin{array}{l}\text { Schizothorax esocinus } \\
\text { (Heckel, 1838) } \\
\text { (Cyprinidae) }\end{array}$ & Chirruh Snow trout & Chhurru & Flesh & $\begin{array}{l}\text { Rista, Kabab, Muj-gad, Nadur-gad, Tikken, Tailth- } \\
\text { gad, Kantii }\end{array}$ & Kashmiri \\
\hline $\begin{array}{l}\text { Schizothorax labiatus } \\
\text { (McClelland, 1842) } \\
\text { (Cyprinidae) }\end{array}$ & Kunar Snow trout & Chush & Flesh & $\begin{array}{l}\text { Rista, Kabab, Muj-gad, Nadur-gad, Tikken, Tailth- } \\
\text { gad, Kantii }\end{array}$ & Kashmiri \\
\hline $\begin{array}{l}\text { Schizothorax plagiostomus } \\
\text { (Heckel, 1838) } \\
\text { (Cyprinidae) }\end{array}$ & Hill trout & Khont & Flesh & $\begin{array}{l}\text { Rista, Kabab, Muj-gad, Nadur-gad, Tikken, Tailth- } \\
\text { gad, Kantii } \\
\text { *Nadur-gad is prepared by cooking fish with } \\
\text { lotus roots. } \\
\text { *Tailth gad is prepared by frying fish in oil. }\end{array}$ & Kashmiri \\
\hline $\begin{array}{l}\text { Streptopelia decaocto } \\
\text { (Frivaldszky, 1838) } \\
\text { (Columbidae) }\end{array}$ & $\begin{array}{ll}\text { Dove, } & \text { Eurasian } \\
\text { Collared } & \end{array}$ & Kukil & $\begin{array}{l}\text { Flesh } \\
\text { Liver } \\
\text { Heart } \\
\text { Legs }\end{array}$ & Shakaar-maaz & Kashmiri \\
\hline $\begin{array}{l}\text { Streptopelia orientalis } \\
\text { (Latham, 1790) } \\
\text { (Columbida e) }\end{array}$ & Oriental turtle dove & Wan Kukil & $\begin{array}{l}\text { Flesh } \\
\text { Liver } \\
\text { Heart } \\
\text { Legs }\end{array}$ & Shakaar-maaz & Kashmiri \\
\hline $\begin{array}{l}\text { Tadorna ferruginea } \\
\text { (Pallas, 1764) } \\
\text { (Anatidae) }\end{array}$ & Shelduck, Ruddy & Surkhab & $\begin{array}{l}\text { Flesh } \\
\text { Liver } \\
\text { Heart } \\
\text { Legs }\end{array}$ & $\begin{array}{l}\text { Shakaar-maaz } \\
\text { *Shakaar-maaz is prepared by cooking or frying. }\end{array}$ & Kashmiri \\
\hline
\end{tabular}


Ethnobotany Research and Applications

\begin{tabular}{|c|c|c|c|c|}
\hline $\begin{array}{l}\text { PLANTS } \\
\text { Scientific Name } \\
\text { (Family) }\end{array}$ & Common Name & Local Name & Recipes & Ethnic Groups \\
\hline $\begin{array}{l}\text { Abelmoschus esculentus (L.) } \\
\text { Moench } \\
\text { (Malvaceae) }\end{array}$ & Lady finger & Bindii & $\begin{array}{l}\text { Bindii } \\
\text { *Bindii is prepared byd cooking of frying the Lady finger. }\end{array}$ & Kashmiri \\
\hline $\begin{array}{l}\text { Agaricus bisporus (J.E. } \\
\text { Lange) Imbach } \\
\text { (Agaricaceae) }\end{array}$ & Mushroom & $\begin{array}{l}\text { Hedar } \\
\text { Hend } \\
\text { Kantrapday }\end{array}$ & $\begin{array}{l}\text { Hedar, Hend } \\
\text { *Hend is prepared by frying or cooking mushroom with } \\
\text { tomato or alone. }\end{array}$ & $\begin{array}{l}\text { Kashmiri } \\
\text { Gujjar } \\
\text { Pahari }\end{array}$ \\
\hline $\begin{array}{l}\text { Allium cepa L. } \\
\text { (Amaryllidaceae) }\end{array}$ & Onion & $\begin{array}{l}\text { Gand } \\
\text { Pyaaz }\end{array}$ & $\begin{array}{l}\text { Rista, Kabab, Gushtab, Rogan-josh, Marchavagun-kurma, } \\
\text { Tabakh-maaz, Yakhin, Aab-gosh, Muj-gad, Tikken, Tailth-gad, } \\
\text { Kantii, Shabdaig, Haak, Nadir, Paalak, Gaguj, Muj, Salaad, } \\
\text { Tamatar, Wangan, Chol-daal, Masour-daal, Muang-daal, } \\
\text { Razma-daal }\end{array}$ & $\begin{array}{l}\text { Kashmiri } \\
\text { Gujjar } \\
\text { Pahari }\end{array}$ \\
\hline $\begin{array}{l}\text { Allium proliferum (Moench) } \\
\text { Schrad. ex Willd } \\
\text { (Amaryllidaceae) }\end{array}$ & Tree onion & $\begin{array}{l}\text { Praan } \\
\text { Praand }\end{array}$ & $\begin{array}{l}\text { Rista, Kabab, Gushtab, Rogan-josh, Marchavagun-kurma, } \\
\text { Yakhin, Aab-gosh, Muj-gad, Nadur-gad,Kantii, Shabdaig, } \\
\text { Nadir, Salaad, Tamatar, Wangan, Chol- daal, Masour-daal, } \\
\text { Muang-daal, Razma-daal }\end{array}$ & $\begin{array}{l}\text { Kashmiri } \\
\text { Gujjar } \\
\text { Pahari }\end{array}$ \\
\hline $\begin{array}{l}\text { Allium sativum } \mathrm{L} \text {. } \\
\text { (Amaryllidaceae) }\end{array}$ & Garlic & $\begin{array}{l}\text { Rohun } \\
\text { Lasan }\end{array}$ & $\begin{array}{l}\text { Rista, Kabab, Gushtab, Rogan-josh, Marchavagun-kurma, } \\
\text { Tabakh-maaz, Yakhin, Aab gosh, Muj Gad, Nadir Gad, Tikken, } \\
\text { Tailth Gad, Kantii, Shabdaig, Nadir, Gaguj, Salaad, Tamatar, } \\
\text { Wangan,Chol-daal, Masour-daal, Muang-daal, Razma-daal }\end{array}$ & $\begin{array}{l}\text { Kashmiri } \\
\text { Gujjar } \\
\text { Pahari }\end{array}$ \\
\hline $\begin{array}{l}\text { Amaranthus retroflexus } \mathrm{L} \text {. } \\
\text { (Amaranthaceae) }\end{array}$ & Redroot pigweed & Ganhaar & $\begin{array}{l}\text { Crai } \\
{ }^{*} \text { Crai is prepared by frying the Redroot pigweed seeds. }\end{array}$ & Kashmiri \\
\hline $\begin{array}{l}\text { Amomum subulatum Roxb. } \\
\text { (Zingiberaceae) }\end{array}$ & Black cardamom & $\begin{array}{l}\text { Bad aal } \\
\text { Bajaal }\end{array}$ & $\begin{array}{l}\text { Rista, Kabab, Gushtab, Rogan-josh, Marchavagun-kurma, } \\
\text { Yakhin, Aab gosh, Muj-gad, Nadir-gad, Tikken, Kantii, } \\
\text { Shabdaig, Nadir, Gaguj, Tamatar, Wangan, Chol-daal, } \\
\text { Masour-daal, Muang-daal, Razma-daal, Pulao }\end{array}$ & $\begin{array}{l}\text { Kashmiri } \\
\text { Gujjar } \\
\text { Pahari }\end{array}$ \\
\hline $\begin{array}{l}\text { Anacardium occidentale L. } \\
\text { (Anacardiaceae) }\end{array}$ & Cashew & Kajoov & Kaju, Salaad, Pulao & Kashmiri \\
\hline $\begin{array}{l}\text { Ananas comosus (L.) Merr } \\
\text { Bromeliaceae) }\end{array}$ & Pineapple & Ananas & Salaad & Kashmiri \\
\hline $\begin{array}{l}\text { Brassica oleracea var. } \\
\text { botrytis } \\
\text { (Brassicaceae) }\end{array}$ & Cauliflower & $\begin{array}{l}\text { Fulgopi } \\
\text { Foolgopi }\end{array}$ & $\begin{array}{l}\text { Fulgopi } \\
\text { * Fulgopi is prepared by cooking Cauliflower. }\end{array}$ & $\begin{array}{l}\text { Kashmiri } \\
\text { Gujjar } \\
\text { Pahari }\end{array}$ \\
\hline $\begin{array}{l}\text { Brassica oleracea var. } \\
\text { capitata } \\
\text { (Brassicaceae) }\end{array}$ & Cabbage & $\begin{array}{l}\text { Band-Haak } \\
\text { Bandgobi }\end{array}$ & $\begin{array}{l}\text { Band-haak } \\
\text { *Band-haak is prepared by cooking cabbage. }\end{array}$ & $\begin{array}{l}\text { Kashmiri } \\
\text { Gujjar } \\
\text { Pahari }\end{array}$ \\
\hline
\end{tabular}


Ethnobotany Research and Applications

\begin{tabular}{|c|c|c|c|c|}
\hline $\begin{array}{l}\text { Brassica rapa var. rapa } \mathrm{L} . \\
\text { (Brassicaceae) }\end{array}$ & Turnip & $\begin{array}{l}\text { Gugaij } \\
\text { Shaljam }\end{array}$ & $\begin{array}{l}\text { Gaguj } \\
\text { Gaguj is prepared by cooking or frying Turnip. }\end{array}$ & $\begin{array}{l}\text { Kashmiri } \\
\text { Gujjar } \\
\text { Pahari }\end{array}$ \\
\hline $\begin{array}{l}\text { Cajanus cajan (L.) Millsp. } \\
\text { (Fabaceae) }\end{array}$ & Red gram & $\begin{array}{l}\text { Masour } \\
\text { Rati daal }\end{array}$ & $\begin{array}{l}\text { Masour-daal } \\
\text { * Masour-daal is prepared by cooking Red gram with some } \\
\text { spices. }\end{array}$ & $\begin{array}{l}\text { Kashmiri } \\
\text { Gujjar } \\
\text { Pahari }\end{array}$ \\
\hline $\begin{array}{l}\text { Capsicum annuum L. } \\
\text { (Solanaceae) }\end{array}$ & Chili peppers & $\begin{array}{l}\text { Marchwanga } \\
\text { Meraich }\end{array}$ & $\begin{array}{l}\text { Rista, Kabab, Rogan-josh, Marchavagun-kurma, Muj-gad, } \\
\text { Nadir Gad, Tikken, Shabdaig, Nadir, Haak, Nadir, Paalak, } \\
\text { Gugaj, Muj, Wangan, Chol-daal, Masour-daal, Muang-daal, } \\
\text { Razma-daal, Aaloo chips }\end{array}$ & $\begin{array}{l}\text { Kashmiri } \\
\text { Pahari }\end{array}$ \\
\hline $\begin{array}{l}\text { Celosia argentea L. } \\
\text { (Amaranthaceae) }\end{array}$ & Cocks comb & Moval & Marchavagun-kurma & Kashmiri \\
\hline $\begin{array}{l}\text { Cicer arietinum L. } \\
\text { (Fabaceae) }\end{array}$ & Bengal gram & Choldaal & $\begin{array}{l}\text { Choldaal } \\
\text { * Chol dall is prepared by cooking Bengal gram with some } \\
\text { spices\#. }\end{array}$ & $\begin{array}{l}\text { Kashmiri } \\
\text { Gujjar } \\
\text { Pahari }\end{array}$ \\
\hline $\begin{array}{l}\text { Citrus aurantïfolia Swingle } \\
\text { (Rutaceae) }\end{array}$ & Lime & Luemb & Salaad & Kashmiri \\
\hline $\begin{array}{l}\text { Citrus limon (L.) Osbeck } \\
\text { (Rutaceae) }\end{array}$ & Lemon & Luemb & Salaad & Kashmiri \\
\hline $\begin{array}{l}\text { Coriandrum sativum L. } \\
\text { (Apiaceae) }\end{array}$ & Coriander & $\begin{array}{l}\text { Daainvaal } \\
\text { Daniya } \\
\text { Daanvaal }\end{array}$ & $\begin{array}{l}\text { Rista, Kabab, Gushtab, Rogan-josh, Marchavagun-kurma, } \\
\text { Yakhin, Aab gosh, Muj-gad, Nadur-gad, Kantii, Shabdaig, } \\
\text { Nadir, Salaad, Tamatar, Wangan, Chol-daal, Masour-daal, } \\
\text { Muang-daal, Razma-daal }\end{array}$ & $\begin{array}{l}\text { Kashmiri } \\
\text { Gujjar } \\
\text { Pahari }\end{array}$ \\
\hline $\begin{array}{l}\text { Crocus sativus L. } \\
\text { (Iridaceae) }\end{array}$ & Saffron & $\begin{array}{l}\text { Zafraan } \\
\text { Kong }\end{array}$ & Khawa, Pulao & Kashmiri \\
\hline $\begin{array}{l}\text { Cucumis sativus L. } \\
\text { (Cucurbitaceae) }\end{array}$ & Cucumber & $\begin{array}{l}\text { Laar } \\
\text { Tradii }\end{array}$ & Salaad & $\begin{array}{l}\text { Kashmiri } \\
\text { Gujjar } \\
\text { Pahari }\end{array}$ \\
\hline $\begin{array}{l}\text { Cucurbita pepo L. } \\
\text { (Cucurbitaceae) }\end{array}$ & Pumpkin & $\begin{array}{l}\text { Kud } \\
\text { Kudoo }\end{array}$ & Shabdaig & $\begin{array}{l}\text { Kashmiri } \\
\text { Gujjar } \\
\text { Pahari }\end{array}$ \\
\hline $\begin{array}{l}\text { Curcuma longa L. } \\
\text { (Zingiberaceae) }\end{array}$ & Turmeric & $\begin{array}{l}\text { Lidder } \\
\text { Haldi }\end{array}$ & $\begin{array}{l}\text { Rista, Kabab, Rogan-josh, Marchavagun-kurma, Muj-gad, } \\
\text { Nadir Gad, Kantii, Shabdaig, Nadir, Paalak, Gugaj, Muj, } \\
\text { Wangan, Chol-daal, Masour-daal, Muang-daal, Razma-daal, } \\
\text { Tamatar, Pulao }\end{array}$ & $\begin{array}{l}\text { Kashmiri } \\
\text { Gujjar } \\
\text { Pahari }\end{array}$ \\
\hline $\begin{array}{l}\text { Daucus carota subsp. sativus } \\
\text { (Hoffm.) Schübl. \& G. } \\
\text { Martens) }\end{array}$ & Carrot & $\begin{array}{l}\text { Gaazar } \\
\text { Gaajar }\end{array}$ & Salaad & $\begin{array}{l}\text { Kashmiri } \\
\text { Gujjar } \\
\text { Pahari }\end{array}$ \\
\hline
\end{tabular}




\begin{tabular}{|c|c|c|c|c|}
\hline (Apiaceae) & & & & \\
\hline $\begin{array}{l}\text { Elettaria cardamomum (L.) } \\
\text { Maton } \\
\text { (Zingiberaceae) }\end{array}$ & $\begin{array}{l}\text { Green or true } \\
\text { cardamom }\end{array}$ & $\begin{array}{l}\text { Sabazaal } \\
\text { Neachaal } \\
\text { Sabazallachi }\end{array}$ & $\begin{array}{l}\text { Rista, Kabab, Gushtab, Rogan-josh, Marchavagun-kurma, } \\
\text { Yakhin, Aab gosh, Muj-gad, Nadir-gad, Tikken, Kantii, } \\
\text { Shabdaig, Nadir }\end{array}$ & $\begin{array}{l}\text { Kashmiri } \\
\text { Gujjar } \\
\text { Pahari }\end{array}$ \\
\hline $\begin{array}{l}\text { Elwendia persica (Boiss.) } \\
\text { Pimenov \& Kljuykov } \\
\text { (Ranunculaceae) }\end{array}$ & Black cumin & $\begin{array}{l}\text { Khruenzueer } \\
\text { Kala zeera }\end{array}$ & Shabdaig & $\begin{array}{l}\text { Kashmiri } \\
\text { Gujjar } \\
\text { Pahari }\end{array}$ \\
\hline $\begin{array}{l}\text { Foeniculum vulgare Mill. } \\
\text { (Apiaceae) }\end{array}$ & Fennel & $\begin{array}{l}\text { Baidyaan } \\
\text { Soonf }\end{array}$ & $\begin{array}{l}\text { Rista, Kabab, Gushtab, Rogan-josh, Marchavagun-kurma, } \\
\text { Yakhin, Aab-gosh, Muj-gad, Nadur-gad, Kantii, Shabdaig, } \\
\text { Tikken, Shabdaig, Tamatar, Wangan, Chol-daal, Masour-daal, } \\
\text { Muang-daal, Razma-daal }\end{array}$ & $\begin{array}{l}\text { Kashmiri } \\
\text { Gujjar } \\
\text { Pahari }\end{array}$ \\
\hline $\begin{array}{l}\text { Juglans regia } \mathrm{L} \text {. } \\
\text { (Juglandaceae) }\end{array}$ & Walnut & $\begin{array}{l}\text { Doon } \\
\text { Khood } \\
\text { Akhroat }\end{array}$ & $\begin{array}{l}\text { Chut, Salaad } \\
\text { * Chut is prepared by grinding walnut with other ingredients } \\
\text { like mint, radish along with salt. }\end{array}$ & $\begin{array}{l}\text { Kashmiri } \\
\text { Gujjar } \\
\text { Pahari }\end{array}$ \\
\hline $\begin{array}{l}\text { Laurus nobilis L. } \\
\text { (Lauraceae) }\end{array}$ & Bay leaf & Taij pat & $\begin{array}{l}\text { Rista, Kabab, Gushtab, Rogan-josh, Marchavagun-kurma, } \\
\text { Yakhin, Aab-gosh, Mu-gad, Nadir Gad, Tikken, Shabdaig, } \\
\text { Pulao }\end{array}$ & Kashmiri \\
\hline $\begin{array}{l}\text { Malva neglecta Wallr. } \\
\text { (Malvaceae) }\end{array}$ & Commom Mallow & Sochal & $\begin{array}{l}\text { Sochal } \\
\text { *Sochal is prepared by cooking or frying. }\end{array}$ & $\begin{array}{l}\text { Kashmiri } \\
\text { Gujjar } \\
\text { Pahari }\end{array}$ \\
\hline $\begin{array}{l}\text { Mentha spicata L. } \\
\text { (Lamiaceae) }\end{array}$ & Mint & $\begin{array}{l}\text { Fadne } \\
\text { Pudne }\end{array}$ & $\begin{array}{l}\text { Yakhin, Aab-gosh, Tailth-gad, Kantii, Shabdaig, Nadir, Salaad, } \\
\text { Tamatar, Raz-madaal }\end{array}$ & $\begin{array}{l}\text { Kashmiri } \\
\text { Gujjar } \\
\text { Pahari }\end{array}$ \\
\hline $\begin{array}{l}\text { Nelumbo nucifera Gaertn. } \\
\text { (Nelumbonaceae) }\end{array}$ & Lotus & Nadur & $\begin{array}{l}\text { Nadur-gad, Nadir } \\
\text { * Nadir is prepared by cooking lotus roots. }\end{array}$ & Kashmiri \\
\hline $\begin{array}{l}\text { Oryza sativa L. } \\
\text { (Poaceae) }\end{array}$ & Rice & $\begin{array}{l}\text { Tumul } \\
\text { Chaval }\end{array}$ & $\begin{array}{l}\text { Baath, Pulao } \\
\text { *Baath is prepared by steaming the rice for } 20 \text { minutes. } \\
\text { *Pulao is prepared by cooking rice with dry fruits, and butter. }\end{array}$ & $\begin{array}{l}\text { Kashmiri } \\
\text { Gujjar } \\
\text { Pahari }\end{array}$ \\
\hline $\begin{array}{l}\text { Phaseolus vulgaris } \mathrm{L} \text {. } \\
\text { (Fabaceae) }\end{array}$ & Common bean & $\begin{array}{l}\text { Razmadaal } \\
\text { Rajma }\end{array}$ & $\begin{array}{l}\text { Razma-daal } \\
\text { * Razma-daal is prepared by cooking the Common bean in } \\
\text { cooker for more than one hour. }\end{array}$ & $\begin{array}{l}\text { Kashmiri } \\
\text { Gujjar } \\
\text { Pahari }\end{array}$ \\
\hline $\begin{array}{l}\text { Piper nigrum L. } \\
\text { (Piperaceae) }\end{array}$ & Black pepper & $\begin{array}{l}\text { Marachh } \\
\text { Kali marich }\end{array}$ & $\begin{array}{l}\text { Rista, Kabab, Gushtab, Rogan-josh, Marchavagun-kurma, } \\
\text { Yakhin, Aab gosh, Muj-gad, Nadir-gad,Tikken, Tailth-gad, } \\
\text { Kantii, Shabdaig, Nadir, Gaguj, Tamatar, Wangan, Chol-daal, } \\
\text { Masour-daal, Muang-daal, Razma-daal, Pulao }\end{array}$ & $\begin{array}{l}\text { Kashmiri } \\
\text { Gujjar } \\
\text { Pahari }\end{array}$ \\
\hline
\end{tabular}


Ethnobotany Research and Applications

\begin{tabular}{|c|c|c|c|c|}
\hline $\begin{array}{l}\text { Prunus dulcis (Mill.) D.A. } \\
\text { Webb } \\
\text { (Rosaceae) }\end{array}$ & Almond & Badam & $\begin{array}{l}\text { Khawa, Salaad, Pulao } \\
\text { *Khawa is prepared by boiling cinnamon in water with other } \\
\text { spices like saffaron for more than half an hour. }\end{array}$ & Kashmiri \\
\hline $\begin{array}{l}\text { Punica granatum L. } \\
\text { (Lythraceae) }\end{array}$ & Pomegranate & $\begin{array}{l}\text { Daen } \\
\text { Aaanar }\end{array}$ & Salaad & $\begin{array}{l}\text { Kashmiri } \\
\text { Gujjar } \\
\text { Pahari }\end{array}$ \\
\hline $\begin{array}{l}\text { Raphanus raphanistrum L. } \\
\text { (Brassicaceae) }\end{array}$ & Radish & $\begin{array}{l}\text { Muj } \\
\text { Mooli }\end{array}$ & Muj-gad, Muj,Salaad & $\begin{array}{l}\text { Kashmiri } \\
\text { Gujjar } \\
\text { Pahari }\end{array}$ \\
\hline $\begin{array}{l}\text { Solanum lycopersicum L. } \\
\text { (Solanaceae) }\end{array}$ & Tomato & $\begin{array}{l}\text { Tamatar } \\
\text { Marvaangun }\end{array}$ & $\begin{array}{l}\text { Rogan-josh, Marchavagun-kurma, Muj-gad, Nadir-gad, } \\
\text { Kantii, Shabdaig, Nadir, Gaguj, Salaad, Paalak, Salaad, } \\
\text { Tamatar, Wangan, Chol-daal,Masour-daal, Muang-daal, } \\
\text { Razma-daal }\end{array}$ & $\begin{array}{l}\text { Kashmiri } \\
\text { Gujjar } \\
\text { Pahari }\end{array}$ \\
\hline $\begin{array}{l}\text { Solanum melongena L. } \\
\text { (Solanaceae) }\end{array}$ & Egg plant & $\begin{array}{l}\text { Wangun } \\
\text { Baingand }\end{array}$ & Wangan & $\begin{array}{l}\text { Kashmiri } \\
\text { Gujjar } \\
\text { Pahari }\end{array}$ \\
\hline $\begin{array}{l}\text { Solanum tuberosum L. } \\
\text { (Solanaceae) }\end{array}$ & Potato & $\begin{array}{l}\text { Aalu } \\
\text { Alaoo }\end{array}$ & $\begin{array}{l}\text { Aaloo chips } \\
\text { * Aaloo chips are prepared by slicing potato then frying } \\
\text { them in oil. }\end{array}$ & $\begin{array}{l}\text { Kashmiri } \\
\text { Gujjar } \\
\text { Pahari }\end{array}$ \\
\hline $\begin{array}{l}\text { Spinacia oleracea L. } \\
\text { (Amaranthaceae) }\end{array}$ & Spinach & Paalak & $\begin{array}{l}\text { Paalak } \\
\text { *Paalak is prepared by frying spinach leaves then cooked. }\end{array}$ & $\begin{array}{l}\text { Kashmiri } \\
\text { Gujjar } \\
\text { Pahari }\end{array}$ \\
\hline $\begin{array}{l}\text { Syzygium aromaticum (L.) } \\
\text { Merr. \& L.M. Perry } \\
\text { (Myrtaceae) }\end{array}$ & Clove & $\begin{array}{l}\text { Rong } \\
\text { Loong }\end{array}$ & $\begin{array}{l}\text { Rista, Kabab, Gushtab, Rogan-josh, Marchavagun-kurma, } \\
\text { Yakhin, Aab-gosh, Muj-gad, Nadir-gad, Tikken, Shabdaig, } \\
\text { Nadir, Wangan, Chol-daal, Masour-daal, Muang-daal, Pulao, } \\
\text { Razma-daal }\end{array}$ & $\begin{array}{l}\text { Kashmiri } \\
\text { Gujjar } \\
\text { Pahari }\end{array}$ \\
\hline $\begin{array}{l}\text { Taraxacum officinale } \\
\text { (L.) Weber ex F.H. Wig } \\
\text { (Asteraceae) }\end{array}$ & Common dandelion & $\begin{array}{l}\text { Hand } \\
\text { Madan } \\
\text { Handri }\end{array}$ & $\begin{array}{l}\text { Hand } \\
\text { *Hand is prepared by simple cooking Common dandelion. }\end{array}$ & $\begin{array}{l}\text { Kashmiri } \\
\text { Gujjar } \\
\text { Pahari }\end{array}$ \\
\hline $\begin{array}{l}\text { Trigonella foenum-graecum } \\
\text { L. } \\
\text { (Fabaceae) }\end{array}$ & Fenugreek & $\begin{array}{l}\text { Meth } \\
\text { Methii }\end{array}$ & $\begin{array}{l}\text { Rogan-josh, Marchavagun-Kurma, Yakhin, Aab-gosh, Muj- } \\
\text { gad, Nadur-gad,kantii, Shabdaig, Muj, Wangan, Chol-daal, } \\
\text { Masour-daal, Muang-daal, Razma-daal, Methi-maaz }\end{array}$ & $\begin{array}{l}\text { Kashmiri } \\
\text { Gujjar } \\
\text { Pahari }\end{array}$ \\
\hline $\begin{array}{l}\text { Triticum aestivum } \mathrm{L} . \\
\text { (Poaceae) }\end{array}$ & Wheat & $\begin{array}{l}\text { Kanek } \\
\text { Kanak }\end{array}$ & $\begin{array}{l}\text { Nesaashtae } \\
\text { *Nesaashtae is prepared by frying wheat extract with } \\
\text { different additives like dry fruits, and gee. }\end{array}$ & $\begin{array}{l}\text { Kashmiri } \\
\text { Gujjar } \\
\text { Pahari }\end{array}$ \\
\hline $\begin{array}{l}\text { Vigna mungo (L.) Hepper } \\
\text { (Fabaceae) }\end{array}$ & Black gram & $\begin{array}{l}\text { Muth } \\
\text { Kali daal }\end{array}$ & $\begin{array}{l}\text { Muth } \\
\text { * Muth is prepared by cooking Black gram along with some } \\
\text { spices. }\end{array}$ & $\begin{array}{l}\text { Kashmiri } \\
\text { Gujjar } \\
\text { Pahari }\end{array}$ \\
\hline
\end{tabular}




\section{Ethnobotany Research and Applications}

\begin{tabular}{|l|l|l|l|}
\hline $\begin{array}{l}\text { Vigna radiata (L.) R. Wilczek } \\
\text { (Fabaceae) }\end{array}$ & Green gram & $\begin{array}{l}\text { Muang } \\
\text { Sabazdaal }\end{array}$ & $\begin{array}{l}\text { Muang-daal } \\
\text { * Muajjar } \\
\text { Pahari } \\
\text { spices.. }\end{array}$ \\
\hline $\begin{array}{l}\text { Zea mays L. } \\
\text { (Poaceae) }\end{array}$ & Maiz & $\begin{array}{l}\text { Vaath } \\
\text { * Vaath is prepared by roasting the cob on lump charcoal. }\end{array}$ \\
\hline $\begin{array}{l}\text { Zingiber officinale Roscoe } \\
\text { (Zingiberaceae) }\end{array}$ & Ginger & $\begin{array}{l}\text { Makaii } \\
\text { Pajijar }\end{array}$ \\
\hline
\end{tabular}

* Short glossary of dishes.

- Ethnic groups Pahari and Gujjar used mostly recipes like Rista, Gushtab, Rogan-josh, Nadir, Gaguj, Muj,Tamatar, Wangan, Chol-daal, Masour-daal, Muang-daal, Razma-daal

- Recipes mentioned in the table are produced by the mixing different amounts of different species mentioned in the table 


\section{Discussion}

The biological and cultural history linked with food plants and animals have been the focus of important investigations carried out around the world in recent decades (Barthel et al. 2013). Ethnic groups are seen to be specific with the food patterns and hence possess a unique identity. In the valley (Kashmir), present study evaluated the use of flora and fauna in cultural food and the ethnic groups (Kashmiri, Gujjar, Pahari) associated with them. Kashmiri were found to have maximum usage of the species fallowed by Pahari and Gujjar, this can be attributed to the dominance, cultural richness, and ethos. The greater similarity between Kashmiri and Pahari can be ascribed to the cultural blend due to different reasons like, cross cultural marriages, urbanization. However, the lowest usage of species by the Gujjar can be explained by that they said ethnic groups mainly remain inhabited in the hilly areas lacking the necessary facilities due to exploitation by geopolitical issues (Warikoo 2000) which resulted in the less diversified culture. Further the cross-cultural usage revealed 46 species were commonly used by all ethnic groups. Gairola et al. (2014) reported on the cross-cultural usage of plant species from Himalayas, similarly Hassan et al. (2021) reported the cross-cultural usage of animal species from northwestern Himalayas. Among all documented species 23 were animals and 52 plants. Singh and Bedi (2018) reported 42 number of plant species for the ethnofood usage in the western Himalayas, and Singh and Sultan (2016) also reported various plants from Kashmir used in cultural food. Similarly, Altaf et al. (2017) reported the use of animals in traditional food in Pakistan and in the other parts of Himalayas

The most commonly used, animal parts used were flesh followed by legs, head and liver. Other parts include kidney, heart, lungs, brain, and fat. Locally different parts were used for different recipes. Altaf et al. (2018) reported that brain, fat and other parts of the animals were used in different traditional recipes in Pakistan. Flesh of Boviade family is used for prime recipes of Wazwan (multi-course meal) like, Kebab, Rogan-josh, Rista (meatballs cooked in paprika-saffron-fennel spice gravy), Marchvangen-kurma, Tabakh-maaz (ribs cooked in ghee) (Rather et al. 2016). Meanwhile, different plant species like collard green, spinach, rice, beans, tomatoes etc. are used, have become an integral part of the culture.

Spices and condiments are the main ingredients of the food, provide flavor and aroma. Different spices include pepper, cinnamon, cardamom (green, black), cumin, clove, fennel, garlic, ginger, mint, turmeric, red chilli etc. Similar spices are used in other parts of the world like Iran as a part of their culture (Karizaki 2016). There is a surge of spice use during cultural occasions (marriages, festivals, funerals). The present study recorded more than 14 principal spices traditionally used during marriage ceremonies.

Some selected foods are served as salad which include, pineapple, lime, cherry, lemon, walnut, cashew nuts, almonds etc. Nuts are grounded and mixed with some other species like mint and coriander forming a paste locally known as "chut/cheteen". Almond, saffron and cashew nuts are also served separately. Almonds are used in the traditional beverage called "kehwa",

The Wazwan is a part of the Kashmiri marriages since ancestral time, but has been modified over times. The prime example can be observed in the marriage ceremonies where varieties of meat balls like Rista, Gushtab are served with novel varieties of meat balls in the form of pear locally called "tang". Likewise, the famous recipe Pulaw was formerly made of simple rice but is now widely modified using basmati rice, and dry fruits (Karizaki 2016). Similarly, fish were originally cooked whole, but now fish meat balls are gaining importance and are used along with meat balls. Fish kebabs are also novel to the culture. Some other varieties of Kebab locally known as Shami-kebab, Chapri $K e b a b$ are made from lamb, cow, and goat in a circular form. With change in time and taste, marinated varieties of chicken have been introduced in wazwan, blended with Kashmiri spices like red chilies as a starter on traim (feast for four persons) of marriage parties Phirni, a milk pudding with dry fruits like cashews, almonds, dried coconut and saffron, is a very famous and popular dessert, being served after a feast. Kashmir is known for different rice varieties, including Kamad and Mushkbudji which are costly and served at special occasions like festivals and marriages.

Within the valley it was also observed that religion played a critical role in food consumption. The people following Hindu and Sikh faith were reluctant to consume recipes with animal ingredients due to religious aspects. Contrary to that Muslims were seen to consume foods irrespective of the source (Animal and Plant).

\section{Conclusions}

The gastronomic results of this study clearly demonstrate that the ethnic people inhabiting in the Kashmir have intricate relation with the flora and fauna. A wide range of food species with multipurpose uses are used during 
cultural events by the inhabitants of the Jammu \& Kashmir Himalayas. The result indicated that the meat and fruits are used as prime in all cultural events. Ethnic group "Kashmiri" have cultural richness and substantial usage of species than Gujjar and Pahari. Meanwhile cultural usage of species is unique to principal recipes. Variation in the usage pattern of recipes across the valley is also visible with Capital city Srinagar being recognized as hot spot. Some areas like Kupwara far away from the city are less influenced by the evolution of the food culture. The results of this study clearly show that bio cultural heritage is still string in Kashmir. The evolution is continuing in the food recipes all over the world, as is in Kashmir, hence in this regard present study will greatly help in the conservation of local endemic culture and ethnic food.

\section{Declarations}

Ethics declaration: All participants provided prior informed consent.

Consent to participate: Any person shown in an image agreed that this image could be published.

Author contributions: $\mathrm{MUH}$ collected data. $\mathrm{SMH}, \mathrm{MUH}$ analyzed and interpreted the data and results. $\mathrm{SMH}, \mathrm{MUH}$ SH, UY, HAJ wrote and HUM, FAY and RWB revised the manuscript. All authors read and approved the final manuscript.

Funding: This study did not receive any funding.

Competing interests: The authors declare that they have no competing interests.

\section{Acknowledgments}

Thanks are due to the local people of Kashmir for sharing the ethno-food information and Cooperating during the surveys and interviews. The authors are thankful to those who directly or indirectly helped us during the study.

\section{References}

Altaf A, Haq SM, Shabnum N, Jan HA. 2021. Comparative assessment of Phyto diversity in Tangmarg Forest division in Kashmir Himalaya, India. Acta Ecologica Sinica. doi: 10.1016/j.chnaes.2021.04.009

Altaf M, Javid A, Umair M, Rasheed KJ, Abbasi AM. 2017. Ethnomedicinal and cultural practices of mammals and birds in the vicinity of river Chenab, Punjab-Pakistan. Journal of Ethnobiology and Ethnomedicine 13:41.

Altaf M, Umair M, Abbasi AR, Muhammad N, Abbasi AM. 2018. Ethnomedicinal applications of animal species by the local communities of Punjab, Pakistan. Journal of Ethnobiology and Ethnomedicine 14:55.

Asif M, Haq SM, Yaqoob U, Hassan M, Jan HA. 2021. A preliminary study on the ethno-traditional medicinal plant usage in tehsil "Karnah" of District Kupwara (Jammu and Kashmir) India. Ethnobotany Research \& Applications 21:1-14.

Barthel S, Crumley C, Svedin U. 2013. Bio-cultural refugia-Safeguarding diversity of practices for food security and biodiversity. Global Environmental Change 23:1142-1152.

Boutaud JJ, Becuţ A, Marinescu A. 2016. Food and culture. Cultural patterns and practices related to food in everyday life. Introduction. International Journal of Reviews and Research in Social Sciences6:1-3.

Fischler C. 1988. Food, self and identity. Social science information27:275-292.

Gairola S, Sharma J, Bedi YS. 2014. A cross-cultural analysis of Jammu, Kashmir and Ladakh (India) medicinal plant use. Journal of Ethnopharmacology 155:925-986.

Haq SM, Calixto ES, Yaqoob U, Ahmed R, Mahmoud AH, Bussmann RW, Mohammed OB, Ahmad K, Abbasi AM. 2020a. Traditional Usage of Wild Fauna among the Local Inhabitants of Ladakh, Trans-Himalayan Region. Animals 10:2317.

Haq SM, Khuroo AA, Malik AH, Rashid I, Ahmad R, Hamid M, Dar GH. 2020. Forest ecosystems of Jammu and Kashmir State. In Biodiversity of the Himalaya: Jammu and Kashmir State (pp. 191-208). Springer, Singapore.

Haq SM, Singh B. 2020. Ethnobotany as a Science of Preserving Traditional Knowledge: Traditional Uses of Wild Medicinal Plants from District Reasi, J\&K (Northwestern Himalaya), India. In Singh, B. (Ed.) Botanical Leads for Drug Discovery, Springer, Singapore.

Haq SM, Yaqoob U, Calixto ES, Rahman IU, Hashem A, Abd Allah EF, Alakeel MA, Alqarawi AA, Abdalla M, Hassan M, Bussmann RW. 2021. Plant Resources Utilization among Different Ethnic Groups of Ladakh in Trans-Himalayan Region. Biology 10:827.

Hassan M, Haq SM, Yakoob U, Altaf M, Bussmann RW. 2021. The ethnic diversities in animal-human interactions in former Jammu and Kashmir State-India. Ethnobotany Research \& Applications 22:1-18.

Karizaki VM. 2016. Ethnic and traditional Iranian rice-based foods. Journal of Ethnic Foods 3:124-134. 
Kaw MA. 2010. Central Asian Contribution to Kashmir's Tradition of Religio-Cultural Pluralism. Central Asiatic Journal 54:237-55.

Khanna S, Bhagat S. 2021. The Effect of Food Neophobia and Motivation on Ethnic Food Consumption Intention: An Empirical Evidence from Jammu Region. International Journal of Hospitality \& Tourism Systems 14.1.

Mir AY, Yaqoob U, Hassan M, Bashir F, Zanit SB, Haq SM, Bussmann RW. 2021. Ethnopharmacology and phenology of high-altitude medicinal plants in Kashmir, Northern Himalaya. Ethnobotany Research and Applications 24 :1-5.

Phasha L, Molelekwa GF, Mokgobu MI, Morodi TJ, Mokoena MM, Mudau LS. 2020. Influence of cultural practices on food waste in South Africa - a review. Journal of Ethnic Foods 7:1-3.

Rathe SA, Masoodi FA, Akhter R. 2016. Ethnic meat products of Kashmiri wazwan: a review. Journal of Ethnic Foods $3: 246-250$.

Reddy S, Anitha M. 2015. Culture and its influence on nutrition and oral health. Biomedical \& Pharmacology Journal 8:613.

Reardon T, Minten B. 2011. Surprised by supermarkets: diffusion of modern food retail in India. Journal of Agribusiness in Developing and Emerging Economies.

Sheikh AM, Shabina. 2011. Wazwan: a multicultural repository. Lang India. Languages in India 11:266e77.

Singh B, Bedi YS. 2018. Eating from raw wild plants in Himalaya: Traditional knowledge documentary on Sheena tribe in Kashmir Indian Journal of Natural Products and Resources (IJNPR)[Formerly Natural Product Radiance 8:269-75.

Singh B, Sultan P, Hassan QP, Gairola S, Bedi YS. 2016. Ethnobotany, traditional knowledge, and diversity of wild edible plants and fungi: a case study in the Bandipora district of Kashmir Himalaya, India. Journal of Herbs Spices and Medicinal Plants 22:247-278.

Snedden C. 2021. The significance of Kashmir and Kashmiri identity in J\&K. In Independent Kashmir, Manchester University Press.

Tey YS, Arsil P, Brindal M, Liew SY, Teoh CT, Terano R. 2018. Personal values underlying ethnic food choice: Meansend evidence for Japanese food. Journal of Ethnic Foods 5:33-39.

Wadhawan SK. 2021. Geoheritage and Potential Geotourism in Geoparks-Indian Perspective. In Global Geographical Heritage, Geoparks and Geotourism, Springer, Singapore.

Warikoo K. 2000. Tribal Gujjars of Jammu \& Kashmir. Himalayan and Central Asian Studies 4.

\section{Appendix}

\section{Questionnaire}

\section{- Demography}

1. Name of the respondent.

2. Gender of the respondent.

3. Age of the respondent.

4. Education qualification of the respondent.

5. Profession of the respondent.

6. Ethnic community of the respondent.

7. Name of Place to which respondent belongs.

- Cultural Food Analysis

1. Name the different culture occasions observed in your community?

2. Name the food recipes consumed at different occasions?

3. Name the ingredients (Species) used for particular recipe?

4. Name the parts of species used for a particular recipe?

5. Name the principle recipe used at different occasions?

6. Name the prime species often used during cultural events? 